\title{
On strongly nonlinear gravity waves in a vertically sheared atmosphere
}

\author{
Part I: Spectral stability of the refracted wave
}

https://doi.org/10.1515/mcwf-2020-0103

Received October 21, 2020; accepted December 14, 2020

\begin{abstract}
We investigate strongly nonlinear stationary gravity waves which experience refraction due to a thin vertical shear layer of horizontal background wind. The velocity amplitude of the waves is of the same order of magnitude as the background flow and hence the self-induced mean flow alters the modulation properties to leading order. In this theoretical study, we show that the stability of such a refracted wave depends on the classical modulation stability criterion for each individual layer, above and below the shearing. Additionally, the stability is conditioned by novel instability criteria providing bounds on the mean-flow horizontal wind and the amplitude of the wave. A necessary condition for instability is that the mean-flow horizontal wind in the upper layer is stronger than the wind in the lower layer.
\end{abstract}

Keywords: Internal gravity waves; Modulation equations; Sheared flow

\section{Introduction}

The importance of gravity waves for the atmospheric dynamics and hence for weather and climate forecasting was established in [6] and [18]. Gravity waves redistribute energy vertically and thereby couple the different layers of the atmosphere $[3,10]$. Usually excited in the troposphere, gravity waves may persist deep into the upper atmospheric layers [11-13]. On their journey through the atmospheric layers they may interact with the mean flow. They exert drag onto the horizontal mean-flow, produce heat when dissipating [2], and induce mixing of tracer constituents such as green-house gases [22]. To this day, many questions regarding the sources, propagation and dissipation of gravity waves are still to be answered.

From a conceptional point of view, three distinct types of theories-each with its own benefits and challenges-may be employed to study these questions. First and foremost, linear wave theory provides the foundation for our understanding of gravity waves. It predicts dispersion, refraction, reflection, wave packet formation etc. as long as the amplitudes of the waves can be considered to be infinitesimally small [5].

For large-amplitude gravity waves, perturbation theory is a versatile and powerful tool leading to weakly nonlinear wave theory. The key idea of weakly nonlinear theory is to expand the linear solution to the fluid dynamical equations in an asymptotic series assuming finite but small amplitude. Corrections of the order of the amplitude squared to the linear model add new phenomena to wave theory such as wave-mean-flow interaction and modulational instabilities [16, 26-29]. For internal gravity waves in the oceans, weakly nonlinear theory is almost always sufficient. However, this is often not the case for atmospheric gravity waves. When excited over mountains, tropospheric gravity waves may have amplitudes of the order of magnitude of

\footnotetext{
${ }^{\star}$ Corresponding Author: Mark Schlutow: Institut für Mathematik, Freie Universität Berlin, Germany, E-mail: mark.schlutow@fu-berlin.de

Georg S. Voelker: Institut für Atmosphäre und Umwelt, Goethe Universität Frankfurt am Main, Germany, E-mail: voelker@iau.uni-frankfurt.de
} 
the background wind. In the stratosphere and higher, such strong amplitudes are rather the rule than the exception [9]. The effect of increasing amplitude with height is due to anelastic amplification which is absent in the ocean and caused by the compressibility of air. The majority of atmospheric gravity waves is excited in the troposphere. When they extend into higher altitudes, they encounter exponentially decreasing background density. Due to energy conservation the waves grow exponentially causing strong amplitudes.

The mathematical description of gravity waves beyond weakly nonlinear theory was pioneered by Grimshaw [14, 15] and further analyzed in [1] and also [24] in terms of modulation equations. In this asymptotic theory the small expansion parameter is the ratio between the scale on which the background changes and the wavelength. There is, however, no further restriction on the velocity amplitude and therefore it is allowed to be of the same order as the background flow in contrast to weakly nonlinear theory where the amplitude itself is the expansion parameter. Hence, we may call Grimshaw's modulation equations a strongly nonlinear wave theory.

The notion of "strongly nonlinear" is stipulated in the following sense. A superposition of solutions from the class of strongly nonlinear waves does not lead to small errors when inserted into the governing equations (the Euler or Navier-Stokes equations) as it is the case for the weakly nonlinear theory. Instead such a superposition generates terms by wave-wave interaction of order unity in the asymptotic limit. However, this notion does not necessarily imply that the nonlinearities in the governing equation, i.e. the advection terms, are bigger than the pressure gradient term, say, in terms of non-dimensional analysis. In fact, the asymptotic expansion of Grimshaw's theory is such that the perturbation advection terms vanish due to the solenoidality of the wind field. Some theoretical investigations on strongly nonlinear effects and their implications with respect to observations and modeling were performed in [23, 25]. In [25], the spectral stability of strongly nonlinear wave packets of finite extent was studied. Among others it was found that, due to very large amplitudes, the velocity of the envelope and the linear group velocity, as given by the derivative of the frequency with respect to wavenumber, do not necessarily coincide anymore. This property has direct implication for the interpretation of measurements. In [23], strongly nonlinear, saturated waves where investigated where it was shown that modulational instabilities may lead to secondary wave generation by direct wave-mean-flow interaction.

The objective of this paper is to investigate the interaction of strongly nonlinear waves with a very thin shear layer. This renders a common situation in the real atmosphere, e.g., when a mountain wave meets the tropospheric jet and gets refracted [7]. In the setting of linear theory, this situation was studied in the seminal work [8] using a layered model. They approximated the height-dependent background by piecewise constant functions. This idea was advanced in [19] for linear wave packets. We will adopt the idea of a layered background atmosphere but for strongly nonlinear waves.

Our main results are necessary and sufficient criteria for the stability of a refracted non-hydrostatic wave in a two-layered background. If the mean-flow horizontal wind in the lower layer is stronger than the wind in the upper layer, then the wave is stable with respect to the modulation equations. If, however, the upperlayer mean-flow horizontal wind is stronger, which typically occurs for a gravity wave entering the jet, the refracted wave becomes unstable if both mean-flow horizontal winds meet particular upper bounds and if the amplitude of the wave is sufficiently large.

This work is structured as follows. In section 2, we will introduce Grimshaw's modulation equations as our governing equations. Section 3 will be dedicated to the strongly nonlinear wave refracted at a discontinuity as a particular solution to the modulation equations. We will provide numerical evidence for the asymptotic consistency of such a wave solution in section 4. The stability of the refracted wave will be analyzed in section 5. We will summarize the results in section 6 and offer some concluding remarks in section 7 .

In an envisaged second part of the paper, we will investigate the wave solution and the stability results numerically by means of Large Eddy Simulations. 


\section{The model equations}

We consider vertically modulated, horizontally periodic, non-hydrostatic, and strongly nonlinear gravity waves in the unbounded $x$ - $z$-plane which we model by Grimshaw's modulation equations $[1,15,24]$,

$$
\begin{aligned}
& \frac{\partial k_{z}}{\partial t}+\frac{\partial \omega}{\partial z}=0 \\
& \rho \frac{\partial a}{\partial t}+\frac{\partial}{\partial z}\left(\hat{\omega}^{\prime} \rho a\right)=0 \\
& \rho \frac{\partial u}{\partial t}+\frac{\partial}{\partial z}\left(\hat{\omega}^{\prime} k_{x} \rho a\right)=0 .
\end{aligned}
$$

This coupled set of equations governs the evolution of vertical wavenumber $k_{z}$, wave action density $\rho a$, and mean-flow horizontal wind $u$. The horizontal wavenumber $k_{x}=K_{x}$ is a constant being, without loss of generality, positive. In general, this assumption might be relaxed to horizontally slowly varying wave fields as in [24]. The extrinsic frequency $\omega=\hat{\omega}+k_{x} u$ is determined by the Doppler-shifted intrinsic frequency which depends on wavenumber due to the dispersion relation for non-hydrostatic waves,

$$
\hat{\omega}=\frac{N k_{x}}{|\boldsymbol{k}|}, \quad|\boldsymbol{k}|=\sqrt{k_{x}^{2}+k_{z}^{2}} .
$$

Its derivative with respect to the vertical wavenumber,

$$
\hat{\omega}^{\prime}=-\frac{N k_{x} k_{z}}{|\boldsymbol{k}|^{3}},
$$

represents the vertical linear group velocity. Two coefficients appear representing the background state of the atmosphere, the Brunt-Väisälä frequency $N$ and the density $\rho$ which are generally functions of altitude, $z$. However, we apply the Boussinesq assumption such that

$$
\rho, N=\text { const. }
$$

In Whitham's modulation theory [29], equation (1a) represents conservation of waves. The second equation (1b) yields conservation of wave action. Finally, (1c) describes the acceleration of mean-flow horizontal momentum due to the convergence of the flux of horizontal pseudo-momentum $k_{x} \rho a$.

\section{The refracted wave solution}

In this section, we construct an analytical solution to the modulation equations reminiscent of a typical mountain wave that encounters a jet at a certain height-e.g. the tropospheric or mesospheric jet-and experiences refraction. This is a classical situation from linear wave theory. In contrast to linear theory however, we will see that the wave modifies the background flow by direct nonlinear interaction resulting in a self-induced mean flow and Doppler shifting.

When multiplying $k_{x}$ to (1b), then subtracting (1c) and integrating we find

$$
u(z, t)=k_{x} a(z, t)+U^{0}(z) .
$$

We also find $U^{0}$ as an integration constant that can be identified as the horizontal background wind, the wind without the occurrence of a wave ( $a=0$ ), and $u$ is really the mean-flow horizontal wind.

Let us consider a vertically sheared background flow being piecewise constant with the discontinuity at $z=0$,

$$
U^{0}(z)= \begin{cases}U_{1}^{0}, & z<0 \\ U_{2}^{0}, & z>0\end{cases}
$$


Then a stationary solution is given by

$$
\left(k_{z}(z, t), a(z, t)\right)^{\mathrm{T}}=\left(K_{z}(z), A(z)\right)^{\mathrm{T}}= \begin{cases}\left(K_{z, 1}, A_{1}\right)^{\mathrm{T}}, & z<0, \\ \left(K_{z, 2}, A_{2}\right)^{\mathrm{T}}, & z>0 .\end{cases}
$$

The mean-flow horizontal wind really becomes a diagnostic variable due to (5),

$$
u(z, t)=U(z)=\left\{\begin{array}{ll}
U_{1}, & z<0, \\
U_{2}, & z>0,
\end{array} \quad U_{j}=K_{x} A_{j}+U_{j}^{0}, \quad j=1,2 .\right.
$$

Here and further on in this investigation, $j=1$ denotes the layer below and $j=2$ the layer above the discontinuity at $z=0$. The constant pieces are determined by integration of (1a) and (1b),

$$
\begin{aligned}
\hat{\omega}\left(K_{z, j}\right)+K_{x} U_{j} & =\text { constant } \\
\hat{\omega}^{\prime}\left(K_{z, j}\right) A_{j} & =\text { constant } .
\end{aligned}
$$

The integration constant in (9a) must be zero as the extrinsic frequency is readily the derivative of the phase function with respect to time. Therefore, a stationary phase requires vanishing frequency. By means of this argument and (2), (9a) can be rearranged to obtain

$$
K_{z, j}=-\sqrt{\frac{N^{2}}{U_{j}^{2}}-K_{x}^{2}}
$$

Equation (9b) provides an interesting physical implication: the vertical wave action flux is invariant throughout the layered atmosphere. And in particular, it is constant crossing the interface of the two layers. It can be shown that this invariance is equivalent to perfect transmission in linear theory ([19] and references therein) where the transmission coefficient is defined as

$$
T C=\frac{K_{z, 2}\left|\mathcal{B}_{2}\right|^{2}}{K_{z, 1}\left|\mathcal{B}_{1}\right|^{2}}=1
$$

where $\mathcal{B}_{j}$ is the buoyancy amplitude being linked to the wave action density via

$$
A_{j}=\frac{2\left|\mathcal{B}_{j}\right|^{2}}{N^{2} \hat{\omega}\left(K_{z, j}\right)} .
$$

And consequently the reflexion coefficient fulfills $R C=1-T C=0$. Therefore, our refracted wave solution is valid in the linear limit. Whether it is also valid for large amplitudes, will be verified in section 4.

We need to revisit (10). In order to ensure internal waves we must find

$$
J_{j} \equiv \frac{N^{2}}{K_{X}^{2} U_{j}^{2}}>1 \quad \text { for all } j=1,2 .
$$

Otherwise the discriminant in (10) is negative corresponding to evanescent or rather external waves, i.e. waves that propagate only in the horizontal direction. Here, we have introduced a new parameter $J_{j}$ which will become convenient in the analysis of section 5 . Please note that the mean-flow horizontal wind $u$ has become a diagnostic variable due to (5). Let us concatenate the prognostic variables of the wave solution into a vector,

$$
\boldsymbol{P}(z)=\left(K_{z}, A\right)^{\mathrm{T}} \text {. }
$$

Before we come to the main part of this paper, the stability of the refracted wave, we will test the asymptotic validity of this solution numerically. 


\section{Asymptotic consistency of the refracted wave solution}

Grimshaw's modulation equations as presented in section 2 (c.f. (1)) are based on Wentzel-Kramers-Brillouin (WKB) theory which assumes a length scale separation between the background variation and the wavelength. In the present context where the vertical shear layer has a discontinuous shape this assumption is naturally violated. The modulation equations are formulated on a spatial scale comparable to the scale height, approximately $10 \mathrm{~km}$, for waves with much smaller vertical wavelengths of around $1 \mathrm{~km}$. If we assume that the thickness of the shear layer is of similar order of magnitude as the wavelength, then the change in mean-flow horizontal velocity appears as a discontinuous jump in the modulation equations. To consolidate the use of the modulation equations in this limit of a background shear layer thickness comparable to the vertical wavelength, we employ numerical simulations of the Euler equations for various refracted wave solutions.

\subsection{The model setup}

For our parameter study we utilize the Large Eddy Simulation code PincFloit in Boussinesq mode with a second-order MUSCL scheme and a MC Flux limiter [20,30]. The time integration follows an explicit thirdorder Runge-Kutta scheme. To test the validity of the strongly nonlinear refracted wave solution, we choose a vertically sheared layer in terms of the mean-flow horizontal wind of the form

$$
U(z)=U_{1}+\frac{1}{2}\left(U_{2}-U_{1}\right) \tanh \left(\frac{z}{h}\right)
$$

where the length scale parameter $h$ controls the shear layer thickness. Furthermore, we consider a stationary wave obeying (9) generated by (15). The shear layer is then mirrored in the vertical with a matching wave phase at the boundaries such that vertical periodic boundary conditions can be applied. For such a setup to be meaningful one has to consider the following constraints.

1. The shear layers and model boundaries must be separated well enough to ensure that the periodic boundary condition is practically fulfilled.

2. The well-known static stability criterion [4] must not be violated, that is the buoyancy amplitude of the wave must satisfy

$$
|\mathcal{B}|=\alpha N^{2} /\left|K_{z}\right|<N^{2} /\left|K_{z}\right|
$$

on the entire domain where we call $\alpha<1$ the normalized amplitude.

3. The phase of the wave defined similarly to (10) must match at the model boundary, that is

$$
\int_{z_{1}}^{z_{2}} K_{z}(z) \mathrm{d} z=2 \pi n, \quad K_{z}(z)=-\sqrt{\frac{N^{2}}{U(z)^{2}}-K_{x}^{2}}
$$

with an arbitrary integer $n \in \mathbb{Z}$ and the model bounds $z_{1}$ and $z_{2}$.

4. The horizontal domain must be characterized by periodic boundary conditions and a domain size being a multiple of the chosen horizontal wavelengths, $\lambda_{x}$. That is

$$
l \lambda_{x}=l \frac{2 \pi}{K_{x}}=x_{2}-x_{1}
$$

where $x_{1}$ and $x_{2}$ are the left and right model bounds and $l$ is an arbitrary integer $l \in \mathbb{Z}$.

In accordance with the scaling assumptions we choose the parameters summarized in table 1 . From these values we find the vertical wavelengths $\lambda_{z, 1}=959 \mathrm{~m}$ below the shear layer and $\lambda_{z, 2}=1654 \mathrm{~m}$ above the shear layer. In order to fully resolve the waves, we choose a vertical resolution of $40 \mathrm{~m}$ and a horizontal resolution of $156 \mathrm{~m}$. The time step is chosen dynamically by means of a Courant-Friedrichs-Lewy criterion. Initial conditions near the shear layer as described in section 3 are depicted in figure 1 . Here we compare the anticipated refracted solution for a narrow shear layer with $h=400 \mathrm{~m} \sim \lambda_{z}$ (figure 1a) to a refracted wave solution with a broad shear layer with $h=4000 \mathrm{~m} \gg \lambda_{z}$ (figure $1 \mathrm{~b}$ ). 
Table 1: Summary of simulation parameters.

\begin{tabular}{l|l|l|l}
\hline parameter & value & parameter & value \\
\hline \hline$\Delta x$ & $156 \mathrm{~m}$ & $\Delta z$ & $40 \mathrm{~m}$ \\
$U_{1}$ & $-1.5 \mathrm{~m} \mathrm{~s}^{-1}$ & $U_{2}$ & $-2.5 \mathrm{~m} \mathrm{~s}^{-1}$ \\
$N$ & $10^{-2} \mathrm{~s}^{-1}$ & $h$ & $0 . .44 \mathrm{~km}$ \\
$x_{1}$ & $0 \mathrm{~km}$ & $x_{2}$ & $10 \mathrm{~km}$ \\
$\lambda_{x}$ & $5 \mathrm{~km}$ & $\alpha_{1}$ & 0.8 \\
\hline
\end{tabular}
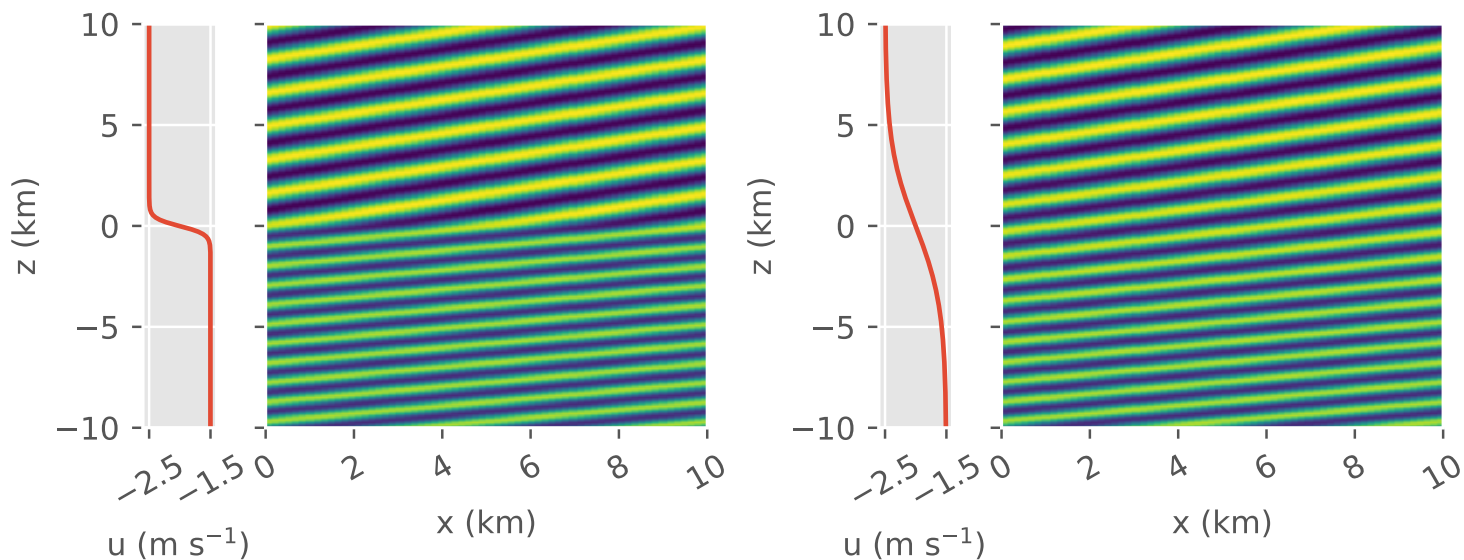

Figure 1: Initial conditions corresponding to the refracted wave solutions of the (WKB) modulation equations near the shear layer for $h=400 \mathrm{~m}$ (a) and $h=4000 \mathrm{~m}$ (b). The color plots show the vertical velocity corresponding to the wave solution as a function of $x$ and $z$. For comparison the shapes of the shear layers are shown.

\subsection{The regime of shear layer thickness comparable to the vertical wavelength}

Before numerically measuring the validity of the refracted wave solution, it is worth noting that Grimshaw's modulation equations (1) are derived under the assumption of the aforementioned scale separation. That is, vertical length scales comparable to the vertical wavelengths, $\lambda_{z}$, are of the order of the small parameter $O(\varepsilon)$ relative to the modulation scales of the mean flow, amplitude and phase. Moreover the solution is valid in the limit $\varepsilon \rightarrow 0$. Hence, variations in the mean-flow horizontal wind on scales similar to the vertical wavelength appear as a discontinuity on the scales of the modulation equations. For the validity of the refracted wave solution (c.f. section 3) near a discontinuous shear layer on the large scale it is therefore sufficient to show numerically that the refracted wave solution is valid in the regime $h=O\left(\lambda_{z}\right)$.

As a diagnostic for the evolution of the numerical simulations we define a relative error $\Delta$ as follows

$$
\Delta(x, t)=\frac{\|w(x, z, t)-w(x, z, 0)\|_{2}}{\|w(x, z, 0)\|_{2}}
$$

where $w(x, z, t)$ is the vertical velocity and $\|f\|_{2}$ is the $L^{2}$-norm defined by

$$
\|f\|_{2}=\left(\int_{-\zeta}^{\zeta} f^{2}(z) \mathrm{d} z\right)^{1 / 2} \quad \text { with } \zeta=10 \mathrm{~km} .
$$

We evaluate $\Delta$ for various $h$ at the horizontal center of the domain, $x=\left(x_{2}-x_{1}\right) / 2$, and at a time $t=3 \mathrm{~h}=$ $10800 \mathrm{~s}$. Note that this time corresponds to the advection time scale given by $1 /\left(K_{x} U\right)=O\left(10^{4} \mathrm{~s}\right)$. We find that $\Delta$ is approximately constant for $h \geq 400 \mathrm{~m}$ at values close to 0.15 (figure 2a). These correspond to a small model error presumably caused by the temporal and spatial discretization schemes of the integration. 

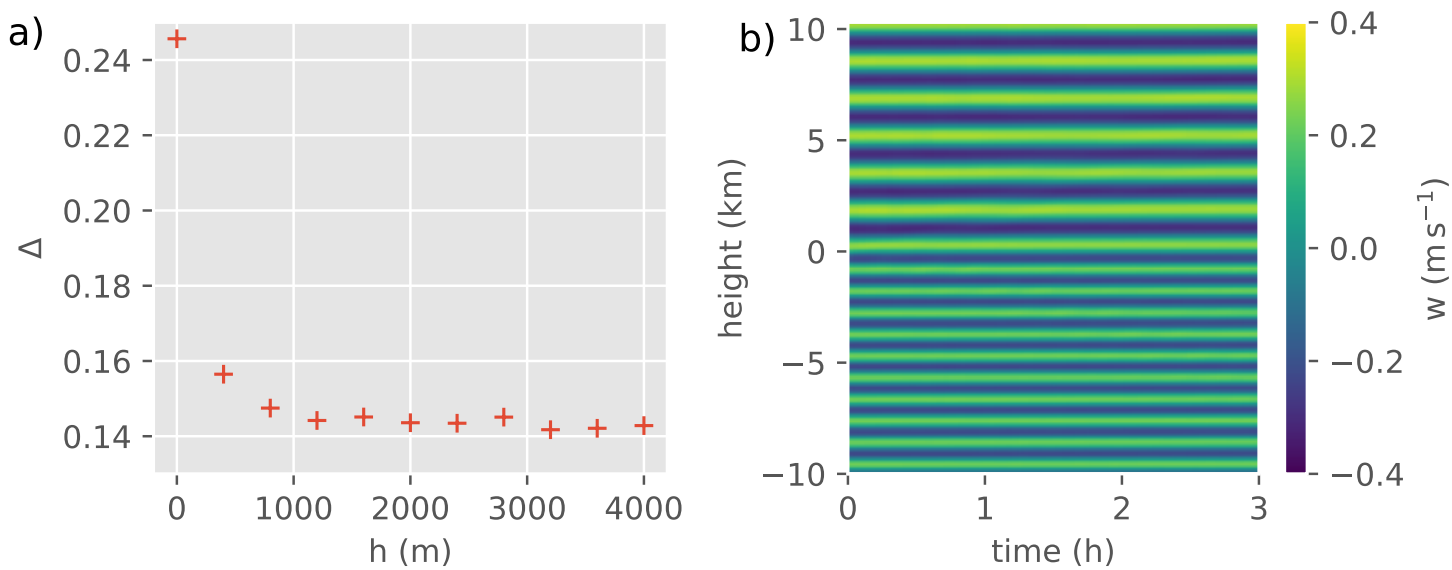

Figure 2: (a) Deviation from the initial condition measured by the relative error norm $\Delta$ as defined in (19) for various values of $h$ and (b) Hovmoeller diagram of the vertical velocity $w(z, t)$ in the horizontal center of the domain.

Only in the extreme case of an exactly discontinuous shear layer we find a $\Delta$ as large as 0.25 associated to an adjustment process of the initial conditions.

Since we have $h=400 \mathrm{~m}<959 \mathrm{~m} \leq \lambda_{z}$, we conclude that the solution suggested in section 3 is asymptotically valid in the regime $h=O\left(\lambda_{z}\right)$. For a qualitative visualization we show a Hovmoeller diagram of the vertical velocity at the horizontal center of the domain for $h=400 \mathrm{~m}$ (figure $2 \mathrm{~b}$ ). We observe that the stationary solution remains visually unperturbed throughout the whole integration even though the normalized amplitude is as large as $\alpha=0.8$. This confirms the hypothesis that the refracted wave solutions are indeed valid for $h=O\left(\lambda_{z}\right)$ even for very large amplitudes.

Summarizing, we have employed numerical simulations of the Euler equations for the proposed refracted wave solutions in the presence of a shear layer with varying thickness, characterized by the length scale $h$. The results confirm that the refracted wave solution is indeed valid in the regime $h=O\left(\lambda_{z}\right)$, not only in the linear regime but also for strongly nonlinear waves with a normalized amplitude as large as $\alpha=0.8$. In particular, we have shown that Grimshaw's modulation equations still apply even for very thin shear layers.

\section{Modulational stability of the refracted wave}

In this section, we will study the stability of the refracted wave solution with regard to the modulation equations. For a more detailed discussion about the perturbation ansatz we refer the interested reader to [25]. Linearizing the governing equations reduced by (5) around the stationary solution $\boldsymbol{P}$ combined with an ansatz for the perturbation

$$
\boldsymbol{p}(z, t)=\tilde{\boldsymbol{p}}(z) e^{\lambda t}, \quad \boldsymbol{p}(z, t)=\left(k_{z}, a\right)^{\mathrm{T}}
$$

yields an eigenvalue problem $(\mathcal{L}-\lambda) \tilde{\boldsymbol{p}}=0$ for a linear differential operator $\mathcal{L}$ which can be reformulated as an ordinary differential equation

$$
\frac{d \tilde{\boldsymbol{p}}(z)}{d z}=\mathbf{B}(z, \lambda) \tilde{\boldsymbol{p}}(z) .
$$

The coefficient matrix B is obtained by inserting ansatz (21) into the governing equations (1) linearized around the wave solution $\boldsymbol{P}$. It contains the refracted wave solution and is therefore piecewise constant in $z$,

$$
\mathbf{B}(z, \lambda)= \begin{cases}\mathbf{B}_{1}, & z<0 \\ \mathbf{B}_{2}, & z>0\end{cases}
$$


where

$$
\begin{aligned}
& \mathbf{B}_{j}=\frac{\lambda}{C_{j}^{2}-K_{x}^{2} A_{j} H_{j}}\left(\begin{array}{cc}
-C_{j} & K_{x}^{2} \\
A_{j} H_{j} & -C_{j}
\end{array}\right), \\
& C_{j}=\hat{\omega}^{\prime}\left(K_{z, j}\right)=-N K_{x} \frac{K_{z, j}}{\left|\boldsymbol{K}_{j}\right|^{3}}, \\
& H_{j}=\hat{\omega}^{\prime \prime}\left(K_{z, j}\right)=N K_{x} \frac{2 K_{z, j}^{2}-K_{x}^{2}}{\left|\boldsymbol{K}_{j}\right|^{5}} .
\end{aligned}
$$

To decide about the stability of our refracted wave solution, we will investigate the spectrum of the linear operator posed by the linearized governing equations. Roughly speaking, the spectrum is the set of all $\lambda \in \mathbb{C}$ for which our operator is not invertible. If the spectrum is contained on the left hand side of the complex plane, i.e. all $\Re(\lambda) \leq 0$, then we can say the solution is spectrally stable and unstable otherwise. The spectrum consists of two qualitatively different subsets defined by its Fredholm properties: the essential (continuous) spectrum and the point (matrix-like) spectrum. We elaborated on this definition in terms of Fredholm operators in the context of strongly nonlinear gravity waves in [25]. For the sake of brevity, the details are not repeated in this paper. We refer to the book of Kapitula and Promislow [17] for a detailed introduction and precise definitions.

\subsection{The essential spectrum}

The essential spectrum is given by the hyperbolicity of the matrix (24) [17, 21]. A matrix is said to be hyperbolic if all its eigenvalues have non-zero real part. The boundaries of the open regions in the complex plane which comprise the essential spectrum coincide exactly with (24) being not hyperbolic. Therefore, the ansatz

$$
\operatorname{det}\left(\mathbf{B}_{j}(\lambda)-i \mu\right)=0, \quad \mu \in \mathbb{R}
$$

leads to the temporal eigenvalues

$$
\lambda_{j}^{ \pm}(\mu)=-i C_{j} \mu \pm i K_{x} \sqrt{A_{j} H_{j}} \mu
$$

in terms of the purely imaginary eigenvalues of $\mathbf{B}_{j}$ which are referred to as the spatial eigenvalues. Equation (28) represents four parameterized curves in the complex plane enclosing the essential spectrum. In particular, we will find unstable essential spectrum if one of the boundary curves enters into the right half of the complex plane as either left or right from the curve we will find essential spectrum. This occurs when $H_{j}<0$ which corresponds to the classical modulational instability criterion [16, 23, 25], i.e

$$
\left|K_{z, j}\right| / K_{x}<1 / \sqrt{2} \text {. }
$$

The instability growth rate is then $K_{x} \sqrt{A_{j}\left|H_{j}\right|}|\mu|$ which happens to be unbounded in $\mu$ or in other words arbitrarily large $\mu$ results in arbitrarily large instability growth which would render the problem unphysical and mathematically ill-posed. The classical modulational stability criterion can be rewritten by insertion of (10) into (26) as

$$
J_{j}>3 / 2
$$

providing us with a new lower bound greater than the criterion for non-evanescence in (13).

\subsection{The point spectrum}

In the following, we assume a stable essential spectrum, so $H_{1}>0$ and $H_{2}>0$. The point spectrum consists of eigenvalues to which the corresponding eigenfunctions belong. Eigenfunctions must be of the form

$$
\tilde{\boldsymbol{p}}=\left\{\begin{array}{ll}
\tilde{\boldsymbol{p}}_{1}, & z<0 \\
\tilde{\boldsymbol{p}}_{2}, & z>0
\end{array} \quad, \quad \tilde{\boldsymbol{p}}_{j}=\hat{\boldsymbol{p}}_{j} e^{\sigma_{j} z}+\mathrm{cc}\right.
$$




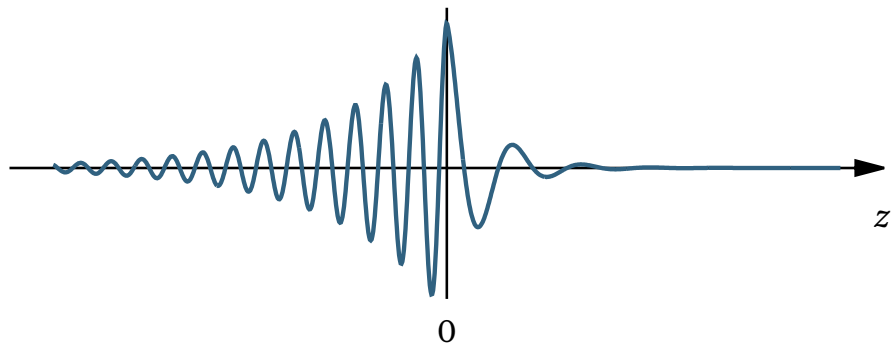

Figure 3: Sketch of the perturbation

with cc denoting the complex conjugate. We obtain the spatial eigenvalues $\sigma_{j}$ by the solvability condition of the emerging algebraic system when inserting the ansatz (31) into (22),

$$
\sigma_{j}^{ \pm}=\frac{\lambda}{ \pm K_{x} \sqrt{A_{j} H_{j}}-C_{j}} .
$$

Please note that \pm indicates two linearly independent solutions. Special care has to be taken for $\pm K_{x} \sqrt{A_{j} H_{j}}=$ $C_{j}$. In this very particular case, $\lambda=0$ is the only eigenvalue. Associated basis vectors to the eigenvalues (32) may be written as

$$
\boldsymbol{q}_{j}^{ \pm}=\left(-K_{x}^{2} \sigma_{j}^{ \pm}, \lambda+C_{j} \sigma_{j}^{ \pm}\right)^{\mathrm{T}} .
$$

In terms of the basis vectors, the eigenvectors are given by superposition, i.e. linear combination,

$$
\tilde{\boldsymbol{p}}_{j}=b_{j}^{+} \boldsymbol{q}_{j}^{+} e^{\sigma_{j}^{+} z}+b_{j}^{-} \boldsymbol{q}_{j}^{-} e^{\sigma_{j}^{-} z}+\mathrm{cc} .
$$

To be an eigenfunction at least one of the coefficients $b_{j}^{ \pm}$must differ from zero. Because if all coefficients vanish, then the solution is trivial and not an eigenfunction. The eigenfunctions must obey three constraints.

1. In the limit $z \rightarrow-\infty$, the eigenfunctions must vanish.

2. In the limit $z \rightarrow+\infty$, the eigenfunctions must vanish.

3. At the discontinuity $z=0$, the eigenfunctions need to be continuous.

These three constraints constitute perturbations of finite energy as well as continuous pressure and vertical wind velocity at the discontinuity of the backround horizontal wind. Figure 3 presents a sketch of the form of the eigenfunctions in line with the constraints $1-3$. They oscillate and decay away from the jump.

Let us fix in the following $\Re(\lambda)>0$ and by this we ask for unstable eigenvalues. We consider (32) and distinguish the four different cases that may occur regarding its sign.

- If $K_{x} \sqrt{A_{1} H_{1}}>C_{1}$, then $\Re\left(\sigma_{1}^{+}\right)>0$ and $\Re\left(\sigma_{1}^{-}\right)<0$. To meet constraint $1, b_{1}^{-}=0$ must therefore be true whereas $b_{1}^{+} \neq 0$ is admissible.

- If $K_{x} \sqrt{A_{1} H_{1}}<C_{1}$, then $\Re\left(\sigma_{1}^{+}\right)<0$ and $\Re\left(\sigma_{1}^{-}\right)<0$. To meet constraint $1, b_{1}^{+}=b_{1}^{-}=0$ must therefore be true.

- If $K_{x} \sqrt{A_{2} H_{2}}>C_{2}$, then $\Re\left(\sigma_{2}^{+}\right)>0$ and $\Re\left(\sigma_{2}^{-}\right)<0$. To meet constraint 2, $b_{2}^{+}=0$ must therefore be true whereas $b_{2}^{-} \neq 0$ is admissible.

- If $K_{x} \sqrt{A_{2} H_{2}}<C_{2}$, then $\Re\left(\sigma_{2}^{+}\right)<0$ and $\Re\left(\sigma_{2}^{-}\right)<0$. Hence, constraint 2 allows for $b_{2}^{+} \neq 0$ and $b_{2}^{-} \neq 0$.

Next, we investigate the continuity constraint 3 , i.e. $\tilde{\boldsymbol{p}}_{1}=\tilde{\boldsymbol{p}}_{2}$ at $z=0$ which yields

$$
b_{1}^{+} \boldsymbol{q}_{1}^{+}=b_{2}^{+} \boldsymbol{q}_{2}^{+}+b_{2}^{-} \boldsymbol{q}_{2}^{-} .
$$

Note that we already anticipated that in all cases $b_{1}^{-}=0$. Given $b_{1}^{+}$we can solve (35) for

$$
\begin{aligned}
& b_{2}^{+}=\frac{1}{2} \frac{+K_{x} \sqrt{A_{2} H_{2}}-C_{2}}{K_{x} \sqrt{A_{1} H_{1}}-C_{1}}\left(1+\sqrt{\frac{H_{1}}{H_{2}}}\right) b_{1}^{+}, \\
& b_{2}^{-}=\frac{1}{2} \frac{-K_{x} \sqrt{A_{2} H_{2}}-C_{2}}{K_{x} \sqrt{A_{1} H_{1}}-C_{1}}\left(1-\sqrt{\frac{H_{1}}{H_{2}}}\right) b_{1}^{+} .
\end{aligned}
$$


We read immediately from (36) that $b_{2}^{+}=b_{2}^{-}=0$ if $b_{1}^{+}=0$ which occurs when $K_{x} \sqrt{A_{1} H_{1}}<C_{1}$ according to constraint 1 . The latter renders therefore a sufficient condition for stability as, if it is true, there exist only trivial solution for any $\Re(\lambda)>0$ and hence it cannot be an eigenvalue. Furthermore, $b_{2}^{+}=0$ fulfilling constraint 2 is only possible if $b_{1}^{+}=0$ due to (36a), but then (36b) yields also $b_{2}^{-}=0$. And again we are left with the trivial solution. By the same argument, $K_{x} \sqrt{A_{2} H_{2}}>C_{2}$ is hence a sufficient condition for stability. In conclusion, there are nontrivial solutions, which happen to be unstable, if

$$
\begin{aligned}
& K_{x} \sqrt{A_{2} H_{2}}<C_{2}, \\
& K_{x} \sqrt{A_{1} H_{1}}>C_{1} .
\end{aligned}
$$

\section{Summary}

In this section, we summarize and reformulate the results of the previous sections. We may rewrite our criterion from the point spectrum in terms of the normalized amplitudes of the waves $\alpha_{j} \in(0,1)$ which relates to the wave action density by

$$
A_{j}=\frac{N^{2}}{2 K_{z, j}^{2} \hat{\omega}\left(K_{z, j}\right)} \alpha_{j}^{2}
$$

when combining (12) and (16).

Thus, by inserting (38) and (13) into the criteria (37) and rearranging by means of (25) and (26), the refracted wave is statically stable but unstable due to perturbations from the point spectrum if

$$
\begin{aligned}
1 & >\alpha_{1}^{2}>\frac{2}{J_{1}} \frac{\left(J_{1}-1\right)^{2}}{2 J_{1}-3}, \\
\frac{2}{J_{2}} \frac{\left(J_{2}-1\right)^{2}}{2 J_{2}-3} & >\alpha_{2}^{2}>0 .
\end{aligned}
$$

To have unity as the upper bound for the right hand side in (39a) implies that $J_{1} \in(2, \infty)$. It represents a particularly narrowing bound on the amplitude in the lower layer as,

$$
\min _{J_{1} \in(2, \infty)} \frac{2}{J_{1}} \frac{\left(J_{1}-1\right)^{2}}{2 J_{1}-3}=\frac{8}{9} \text {. }
$$

The minimum is assumed at $J_{1}=3$, so at best $1>\alpha_{1}>\sqrt{8 / 9} \approx 0.94$. Zero as the lower bound for the left hand side in (39b) is met if $J_{2}>3 / 2$ which is the same bound from the stable essential spectrum.

Utilizing the fact that the vertical wave action flux is invariant across the interface (cf. (9b)), we find $C_{1} A_{1}=C_{2} A_{2}$. We combine our finding with (38) and obtain

$$
\alpha_{1}^{2}=\sqrt{\frac{J_{1}-1}{J_{2}-1}} \frac{J_{1}}{J_{2}} \alpha_{2}^{2}
$$

which we substitute in (39). The resulting inequality reads

$$
\frac{1}{J_{2}^{2}} \frac{\left(J_{2}-1\right)^{3 / 2}}{2 J_{2}-3}>\frac{1}{J_{1}^{2}} \frac{\left(J_{1}-1\right)^{3 / 2}}{2 J_{1}-3}
$$

being the same as $g\left(J_{2}\right)>g\left(J_{1}\right)$. Notice that the function $g$ is continuous as well as monotonically decreasing in $(3 / 2, \infty)$ and hence

$$
J_{2}<J_{1}
$$

which implies due to (13) that $\left|U_{1}\right|<\left|U_{2}\right|$. 
In conclusion, a refracted gravity wave, that is statically stable, non-evanescent, non-hydrostatic and features a stable essential spectrum of the linearized modulation equations, becomes unstable due to perturbations from the point spectrum if

$$
\left|U_{1}\right|<\frac{1}{\sqrt{2}} \frac{N}{K_{x}}, \quad\left|U_{2}\right|<\sqrt{\frac{2}{3}} \frac{N}{K_{x}}, \quad\left|U_{1}\right|<\left|U_{2}\right|
$$

and

$$
\alpha_{1}^{2}>\frac{2}{J_{1}} \frac{\left(J_{1}-1\right)^{2}}{2 J_{1}-3}, \quad J_{1}=\frac{N^{2}}{K_{\chi}^{2} U_{1}^{2}}
$$

\section{Discussion}

On the one hand, the constraint on the normalized amplitude in the lower layer for an emerging instability is extreme. It is at best $94 \%$ of the saturation amplitude or higher. On the other hand, the conditions for the mean-flow horizontal wind favorable for instability are fairly common. A typical situation where this type of instability may occur is the lower edge of the atmospheric jets where a mountain wave enters a strong shear zone from below and gets refracted into the jet. It is not unlikely that such a wave has large amplitude due to anelastic amplification.

The perturbation affects not only the wave properties but also the mean-flow horizontal wind as a diagnostic variable due to (5). Also we observe that perturbations decay exponentially away from the discontinuity of the background wind (cf. figure 3 for illustration). We conclude therefore that a point-spectrum instability is localized at the jump. Due to these observations we can suspect an attenuation of the sharp gradient in the background horizontal wind by an instability from the point spectrum. In conclusion, strongly nonlinear gravity waves may serve as a mitigation of strongly sheared flows.

By means of Large Eddy Simulations we will investigate the dynamics of the point-spectrum instabilities at the shear layer in a successive paper where we will also explore in detail how this mechanism competes with other known instability processes.

Acknowledgments: This research was supported by the German Research Foundation (DFG) through the Research Unit FOR 1898 (MS-GWaves), through grants KL 611/25-2, AC 71/10-2 and BO 5071/1-2.

\section{References}

[1] Ulrich Achatz, R. Klein, and F. Senf. Gravity waves, scale asymptotics and the pseudo-incompressible equations. Journal of Fluid Mechanics, 663:120-147, 2010. 10.1017/S0022112010003411.

[2] Erich Becker. Direct heating rates associated with gravity wave saturation. Journal of Atmospheric and Solar-Terrestrial Physics, 66(6-9):683-696, 2004. 10.1016/j.jastp.2004.01.019.

[3] Erich Becker. Dynamical control of the middle atmosphere. Space Science Reviews, 168(1-4):283-314, 2012. 10.1007/s11214-011-9841-5.

[4] Gergely Bölöni, Bruno Ribstein, Jewgenija Muraschko, Christine Sgoff, Junhong Wei, and Ulrich Achatz. The interaction between atmospheric gravity waves and large-scale flows: An efficient description beyond the nonacceleration paradigm. Journal of the Atmospheric Sciences, 73(12):4833-4852, aug 2016. 10.1175/JAS-D-16-0069.1.

[5] F Bretherton. The propagation of groups of internal gravity waves in a shear flow. Quarterly Journal of the Royal Meteorological Society, 92(394):466-480, 1966. 10.1002/qj.49709239403.

[6] Timothy Dunkerton. On the Mean Meridional Mass Motions of the Stratosphere and Mesosphere. Journal of the Atmospheric Sciences, 35(12):2325-2333, dec 1978. 10.1175/1520-0469(1978)035<2325:0TMMMM>2.0.CO;2.

[7] Benedikt Ehard, Bernd Kaifler, Andreas Dörnbrack, Peter Preusse, Stephen D. Eckermann, Martina Bramberger, Sonja Gisinger, Natalie Kaifler, Ben Liley, Johannes Wagner, and Markus Rapp. Horizontal propagation of large-amplitude mountain waves into the polar night jet. Journal of Geophysical Research, 122(3):1423-1436, 2017. 10.1002/2016JD025621.

[8] Arnt Eliassen and Enok Palm. On the Transfer of Energy in Stationary Mountain Waves. Geofysiske Publikasjoner, 22:1-23, 1961. 
[9] Manfred Ern, Quang Thai Trinh, Peter Preusse, John C. Gille, Martin G. Mlynczak, James M. Russell III, and Martin Riese. GRACILE: a comprehensive climatology of atmospheric gravity wave parameters based on satellite limb soundings. Earth System Science Data, 10(2):857-892, apr 2018. 10.5194/essd-10-857-2018.

[10] David C. Fritts and M Joan Alexander. Gravity wave dynamics and effects in the middle atmosphere. Reviews of Geophysics, 41(1):1003, 2003. 10.1029/2001RG000106.

[11] David C. Fritts, Ronald B. Smith, Michael J. Taylor, James D. Doyle, Stephen D. Eckermann, Andreas Dörnbrack, Markus Rapp, Bifford P. Williams, P.-Dominique Pautet, Katrina Bossert, Neal R. Criddle, Carolyn A. Reynolds, P. Alex Reinecke, Michael Uddstrom, Michael J. Revell, Richard Turner, Bernd Kaifler, Johannes S. Wagner, Tyler Mixa, Christopher G. Kruse, Alison D. Nugent, Campbell D. Watson, Sonja Gisinger, Steven M. Smith, Ruth S. Lieberman, Brian Laughman, James J. Moore, William 0. Brown, Julie A. Haggerty, Alison Rockwell, Gregory J. Stossmeister, Steven F. Williams, Gonzalo Hernandez, Damian J. Murphy, Andrew R. Klekociuk, lain M. Reid, and Jun Ma. The Deep Propagating Gravity Wave Experiment (DEEPWAVE): An Airborne and Ground-Based Exploration of Gravity Wave Propagation and Effects from Their Sources throughout the Lower and Middle Atmosphere. Bulletin of the American Meteorological Society, 97(3):425-453, mar 2016. 10.1175/BAMS-D-1400269.1.

[12] David C Fritts, Simon B Vosper, Bifford P Williams, Katrina Bossert, John M. C. Plane, Michael J Taylor, P.-Dominique Pautet, Stephen D Eckermann, Christopher G Kruse, Ronald B Smith, Andreas Dörnbrack, Markus Rapp, Tyler Mixa, lain M Reid, and Damian J Murphy. Large-Amplitude Mountain Waves in the Mesosphere Accompanying Weak Cross-Mountain Flow During DEEPWAVE Research Flight RF22. Journal of Geophysical Research: Atmospheres, 123(18):9992-10022, sep 2018. 10.1029/2017JD028250.

[13] David C. Fritts, Ling Wang, Michael J. Taylor, Pierre Dominique Pautet, Neal R. Criddle, Bernd Kaifler, Stephen D. Eckermann, and Ben Liley. Large-Amplitude Mountain Waves in the Mesosphere Observed on 21 June 2014 During DEEPWAVE: 2. Nonlinear Dynamics, Wave Breaking, and Instabilities. Journal of Geophysical Research: Atmospheres, 124(17-18):10006-10032, sep 2019. 10.1029/2019JD030899.

[14] R. Grimshaw. Nonlinear internal gravity waves in a slowly varying medium. Journal of Fluid Mechanics, 54(2):193-207, 1972. $10.1017 /$ S0022112072000631.

[15] R. Grimshaw. Internal gravity waves in a slowly varying, dissipative medium. Geophysical Fluid Dynamics, 6:131-148, 1974. $10.1080 / 03091927409365792$.

[16] R. Grimshaw. The Modulation of an Internal Gravity-Wave Packet, and the Resonance with the Mean Motion. Studies in Applied Mathematics, 56(3):241-266, jun 1977. 10.1002/sapm1977563241.

[17] Todd Kapitula and Keith Promislow. Spectral and Dynamical Stability of Nonlinear Waves, volume 185 of Applied Mathematical Sciences. Springer New York, New York, NY, 2013. ISBN 978-1-4614-6994-0. 10.1007/978-1-4614-6995-7.

[18] R. S. Lindzen. Turbulence and stress owing to gravity wave and tidal breakdown. Journal of Geophysical Research, 86(C10): 9707-9714, oct 1981. 10.1029/JC086iC10p09707.

[19] Christopher Pütz, Mark Schlutow, and Rupert Klein. Initiation of ray tracing models: evolution of small-amplitude gravity wave packets in non-uniform background. Theoretical and Computational Fluid Dynamics, 33(5):509-535, oct 2019. 10.1007/s00162-019-00504-z.

[20] Felix Rieper, Stefan Hickel, and Ulrich Achatz. A Conservative Integration of the Pseudo-Incompressible Equations with Implicit Turbulence Parameterization. Monthly Weather Review, 141(3):861-886, 2013. 10.1175/MWR-D-12-00026.1.

[21] Björn Sandstede. Stability of travelling waves. In Bernold Fiedler, editor, Handbook of dynamical systems, volume 2, pages 983-1055. Gulf Professional Publishing, 2002. ISBN 978-0444826695.

[22] M Schlutow, E Becker, and H Körnich. Positive definite and mass conserving tracer transport in spectral GCMs. Journal of Geophysical Research: Atmospheres, 119(20):11,511-562,577, 2014. 10.1002/2014JD021661.

[23] Mark Schlutow. Modulational Stability of Nonlinear Saturated Gravity Waves. Journal of the Atmospheric Sciences, 76(11): 3327-3336, nov 2019. 10.1175/JAS-D-19-0065.1.

[24] Mark Schlutow, R. Klein, and U. Achatz. Finite-amplitude gravity waves in the atmosphere: travelling wave solutions. Journal of Fluid Mechanics, 826:1034-1065, sep 2017. 10.1017/jfm.2017.459.

[25] Mark Schlutow, Erik Wahlén, and Philipp Birken. Spectral stability of nonlinear gravity waves in the atmosphere. Mathematics of Climate and Weather Forecasting, 5(1):12-33, 2019. 10.1515/mcwf-2019-0002.

[26] Bruce R. Sutherland. Finite-amplitude internal wavepacket dispersion and breaking. Journal of Fluid Mechanics, 429:343380, feb 2001. 10.1017/S0022112000002846.

[27] Bruce R. Sutherland. Weakly nonlinear internal gravity wavepackets. Journal of Fluid Mechanics, 569:249-258, 2006. $10.1017 /$ S0022112006003016.

[28] Ali Tabaei and T. R. Akylas. Resonant long-short wave interactions in an unbounded rotating stratified fluid. Studies in Applied Mathematics, 119(3):271-296, 2007. 10.1111/j.1467-9590.2007.00389.x.

[29] G. B. Whitham. Linear and Nonlinear Waves. John Wiley \& Sons, Inc., 1974. ISBN 0471940909. 10.1002/9781118032954.

[30] Jannik Wilhelm, T. R. Akylas, Gergely Bölöni, Junhong Wei, Bruno Ribstein, Rupert Klein, and Ulrich Achatz. Interactions between Mesoscale and Submesoscale Gravity Waves and Their Efficient Representation in Mesoscale-Resolving Models. Journal of the Atmospheric Sciences, 75:2257-2280, 2018. 10.1175/JAS-D-17-0289.1. 